Doc Number:

TM-2359-AD

Beams-doc-2426

Version:

Category:

Note

\title{
MARS15 Calculations for MI8 Collimator Design*
}

\author{
Nikolai V. Mokhov and Bruce C. Brown \\ Fermi National Accelerator Laboratory \\ P.O. Box 500, Batavia, Illinois 60510
}

31 October 2006

\section{Contents}

1 Introduction 2

2 Strategy, Input Parameters and Design Criteria 2

3 Earlier MARS Studies 3

4 Using Marble to Reduce Activation 3

5 Energy Deposition $\quad 7$

6 Radiation Fields in Collimator Region $\quad 10$

7 Sump Water Contamination $\quad 12$

8 Conclusions $\quad 12$

\begin{abstract}
A collimation system for the Booster to Main Injector transfer line (MI8 line) has been designed and installed at MI-836 and MI-838. This system removes particles in the beam halo from a $8 \mathrm{GeV}$ Booster beam before it arrives at the Main Injector. A pair of collimators, with horizontal and vertical motion, is installed between the gradient magnets in half-cell 836 and an identical system is placed one cell away (about $90^{\circ}$ in phase advance) at 838. Energy deposition and radiation calculations have been performed for this system with the MARS 15 Monte-Carlo code and are reported in this note. Losses of a Booster beam in each collimator pair are limited to about $1 \%$ of the Booster intensity of $5 \times 10^{12}$ at $10 \mathrm{~Hz}$ by considerations of surface water contamination and hands-on maintenance criteria. Other radiation effects are less restrictive. This note provides input for radiation safety and thermal analyses for these collimators.
\end{abstract}

\footnotetext{
${ }^{*}$ Work supported by the Universities Research Association, Inc., under contract DE-AC02-76CH03000 with the U. S. Department of Energy.
} 


\section{Introduction}

In response to the need to increase the intensity of proton beams in the Main Injector while maintaining residual radiation levels which permit hands-on maintenance, a program to provide collimation in the Main Injector ring and in the MI8 transfer line from the Booster to the Main Injector is being implemented. Studies of the residual radiation in the Main Injector[1] which began in 2004, prior to high intensity operation for the NuMI facility, observed many locations where residual radiation levels were due to very minor restrictions in the available vertical aperture. In order to reduce the number of such locations, a collimation system for the Booster beam delivered to the Main Injector was designed and implemented[2],[3]. The collimators were installed during the Spring 2006 Fermilab Facility Shutdown. Following modifications of the original proposal made in response to deficiencies identified in initial MARS simulations[4], the final design was proposed and the system reviewed in preparation for the mechanical design. Massive optimization simulations of the radiation produced by losses in these collimators were carried out using the MARS 15 Monte-Carlo code[5]. This note describes these studies, and reports results for the optimized design for use in radiation safety and thermal analyses.

\section{Strategy, Input Parameters and Design Criteria}

The beam loss control requirements for the Main Injector demand a collimation system which localizes a substantial beam loss inside of a system whose motion control can be maintained or repaired without excessive radiation exposure. Such a problem has been addressed for the Fermilab Booster[6] where collimation localizes losses from a beam of $5 \times 10^{12}$ protons created at $10 \mathrm{~Hz}$. This is quite unlike the beam collimation for the Fermilab Tevatron where one localizes losses from a beam of $10^{13}$ protons and anti-protons which are re-injected only after hours[7]. The radiation induced in the Tevatron systems is much lower than for the injector machines. Therefore, the MI8 collimator design efforts looked to the Booster design in which a massive shield surrounds the point where lost protons strike the collimators and the radiation is produced. The collimator and its shield are then mounted on a motion system to provide position control. Since collimation in a beamline requires collimation on four sides of the beam at two locations if all large emittance particles are to be removed, simplifications were sought. The particle trajectory of the one pass beam can be more easily controlled than for the Booster, so, the collimators are aligned parallel to the beam horizontally and vertically and no pitch or yaw control is assumed. But since four collimators are required, minimizing the weight to be moved was important.

The 5.2-m long section of MI-8 between $836 \mathrm{~B}$ and $837 \mathrm{~A}$ magnets is a natural choice. For horizontal collimation performed downstream of $836 \mathrm{~B}, \beta_{x}=35 \mathrm{~m}$ and for a $20 \pi$-mm-mrad emittance, the $3.72 \sigma_{x}$ beam size (about $99.9 \%$ of a Gaussian beam) is $13 \mathrm{~mm}$ while the $3.03 \sigma_{x}$ (about $99 \%$ of beam) is $10.65 \mathrm{~mm}$. At this location, $\beta_{y}=20 \mathrm{~m}$ so the beam size is $30 \%$ smaller making vertical collimation at this location straightforward but slightly less desirable. For vertical collimation upstream of 837A, the size ratio is inverted since $\beta_{y}=35 \mathrm{~m}$, the above beam size description applies to the vertical. This location is $90^{\circ}$ of phase advance (plus many $2 \pi$ ) from the MP02 location.

The scraping fractions are determined by an assumed Gaussian beam transverse distribution. A beam density distribution is assumed to be $1 / r$ in the halo region, e.g. $3.72 \sigma<r<10 \sigma$.

The beam power at $8 \mathrm{GeV}$ with $5 \times 10^{12} \mathrm{ppp}$ at $10 \mathrm{~Hz}$ is $64 \mathrm{~kW}$. That corresponds to $5 \times 10^{10} \mathrm{p} / \mathrm{s}$ or $64 \mathrm{~W}$ of the average scraping rate on the collimators for the $0.1 \%$ beam loss scenario, and $5 \times 10^{11} \mathrm{p} / \mathrm{s}$ or $640 \mathrm{~W}$ for the $1 \%$ scenario. Without shielding, corresponding prompt and residual radiation levels inside the tunnel, in ground/sump water and above shielding would exceed the 
regulatory limits:

- Residual dose rate $P_{\gamma}<100 \mathrm{mrem} / \mathrm{hr}=1 \mathrm{mSv} / \mathrm{hr}$ at 1 foot in tunnel (30-day irradiation and 1-day cooling) - hands-on maintenance.

- Prompt dose equivalent in non-controlled areas is $D E<0.05 \mathrm{mrem} / \mathrm{hr}$ at normal operation and $<1 \mathrm{mrem} / \mathrm{hr}$ for the worst case due to accidents; it is $D E<5 \mathrm{mrem} / \mathrm{hr}=0.05 \mathrm{mSv} / \mathrm{hr}$ for limited access areas (needed 13.5 feet of dirt above the tunnel with Booster design).

- Sump water activation: star density averaged over the region where $99 \%$ of stars (nuclear inelastic interactions above $50 \mathrm{MeV}$ ) contained is $\langle S\rangle_{\text {gravel }}<4000 \mathrm{~cm}^{-3} \mathrm{~s}^{-1}$.

Other design considerations include energy deposition in collimators and heat removal; accumulated absorbed dose in cables, motors and instrumentation limiting their lifetime; and air activation issues.

Calculated with MARS15 are 3-D distributions of the contact residual dose rate (mSv/hr) after 30-day continuous irradiation and 1-day cooling, particle fluxes, energy deposition, and the yearly absorbed dose in Gy/yr at at $2 \times 10^{7}$ operational seconds per year. $1 \mathrm{mSv} / \mathrm{hr}=100 \mathrm{mrem} / \mathrm{hr}$, and $1 \mathrm{~Gy}=100 \mathrm{rad}$. Cutoff energy $E_{t h}=0.1 \mathrm{MeV}$.

\section{Earlier MARS Studies}

First consideration in the MARS15 modeling was given to Max Palmer collimators for the $3.72 \sigma$ case[4]. A 8-GeV proton beam with the RMS spot size $\sigma_{x}$ and $\sigma_{y}$ of $3.5 \mathrm{~mm}$ at $\mathrm{CH}$ and $\mathrm{CV}$, respectively, hits the collimators opened $\pm 13 \mathrm{~mm}$ horizontally $(\mathrm{CH})$ and vertically $(\mathrm{CV})$. A horizontal collimator $(\mathrm{CH})$ consisting of a 4-foot Max Palmer Collimator is placed $0.75 \mathrm{~m}$ downstream of the magnet 836B. Setting this collimator to cut beam at $3.72 \sigma_{x}$ will remove both edges of the beam at this size. A vertical collimator $(\mathrm{CV})$ consisting of a 6-foot Max Palmer Collimator is placed $0.75 \mathrm{~m}$ upstream of the magnet 837A. The collimator steel jaws are $14.6 \times 14.6 \mathrm{~cm}$ encapsulated into a 0.6-cm thick steel vessel. A simple model for the 837A magnet is implemented. Components are connected with 6-inch OD, $1.5 \mathrm{~mm}$ thick beam pipes. The conclusion from these studies was that we can do a reasonable job for $0.1 \%$ scraping, but need a local shield of about $40-\mathrm{cm}$ of steel around the Max Palmer Collimators to appropriately reduce residual dose and protect sump water, cables and motors[4]. A higher scraping rate seems to be unrealistic with this design.

As a second possibility we looked at the Booster collimator design[6] for $1 \%$ scraping. We found that setting collimator jaws to cut the beam at $3.03 \sigma_{x}$ with $1 / r$ halo in the $3.03 \sigma<r<10 \sigma$ region $\left(5 \times 10^{11} \mathrm{p} / \mathrm{s}\right)$ require $44 \times 44 \times 44$ inch steel collimators $( \pm 55 \mathrm{~cm})$. The residual dose is $\mathrm{OK}$, but each collimator weighs 14 tons.

\section{Using Marble to Reduce Activation}

Dirt shielding above the MI-8 collimation region is 23 feet compared to 13.5 feet in the Booster case, therefore the prompt dose on the surface is not a driving issue. The groundwater activation in this region is not an issue, while sump water contamination can be reduced with thick shielding considered. At that stage of design, the driver was residual dose rate on the collimator shielding outer surface (to be kept below $1 \mathrm{mSv} / \mathrm{hr}$ or $100 \mathrm{mrem} / \mathrm{hr}$ ).

The lowest activation in materials used in similar cases is found in marble $\left(\mathrm{CaCO}_{3}, \rho=2.7 \mathrm{gcc}\right)$. It was considered for the SSC collimators and dumps and will be used for the LHC beam dumps. It 
was considered for the Booster collimators but prompt dose, residual dose and sump water activation criteria were equally important requiring massive steel shielding anyway. The idea is to replace outer layer of steel shielding with a marble shell. We have found that its optimal thickness is about 5 inches: there is negligible activation in marble itself and it provides $1 / 10$ attenuation for $1-\mathrm{MeV}$ photons leaking from the hot steel core.

However, one needs to be sure that impurities in the material do not become so radioactive as to negate these advantages. Fortunately, the successful bidder for the MI8 collimator marble procured a supply from Carrara (Italy) where the properties are well documented. Table 1 from Reference [8] show the composition. None of the impurities listed at such a level would deteriorate the marble properties exploited here.

Table 1: Carrara Marble average composition

\begin{tabular}{|l|c|c|}
\hline $\mathrm{CaCO}_{3}$ & $\%$ & $>98$ \\
\hline Dolomite & $\%$ & 1.76 \\
\hline $\mathrm{MgO}$ & $\%$ mol. & 1.32 \\
\hline $\mathrm{SiO}_{2}$ & $\%$ & 0.71 \\
\hline $\mathrm{Sr}$ & $\mathrm{ppm}$ & $114-160$ \\
\hline Residual & $\%$ & 1.37 \\
\hline
\end{tabular}

At the first stage we considered the following two cases in MARS 15 simulations: (1) outer $10 \mathrm{~cm}$ of iron replaced with marble ( $\pm 45 \mathrm{~cm}$ iron $+10 \mathrm{~cm}$ marble); (2) iron reduced laterally by $15 \mathrm{~cm}$ on each side followed by $1 \mathrm{~cm}$ marble $( \pm 30 \mathrm{~cm}$ iron $+10 \mathrm{~cm}$ marble). Result was a drastic improvement in shielding performance with respect to residual radiation levels. Then, further optimizations have been performed. As a result, we have arrived at the following configuration (Fig. 1, see [3] for details):

- To better protect downstream magnets reduce collimator aperture from the Booster's $3 \times 3$ inches to $2 \times 2$ inches. Install a marble mask in front of the 837A magnet.

- Do horizontal and vertical scraping on both $\mathrm{CH}$ and $\mathrm{CV}$ : cut beam at $3.03 \sigma$ horizontally and vertically by, e.g., bottom-left corner of the first collimator, and top-right corner of the second collimator.

- Increase stainless steel jaw thickness from $1 \mathrm{~cm}$ to $2 \mathrm{~cm}$ and length from 40 to 45 inches (including a 8-inch taper at the upstream ends).

- Make shielding wider horizontally to provide better protection in aisle..

- Make shielding longitudinally: 5-inch marble +35-inch iron + 5-inch marble.

- Make shielding laterally (on each side): 10-inch iron + 5-inch marble.

As shown in Fig. 2, residual dose rates in this design are safely below the limits except at two locations. On the protruding stainless steel vacuum liner is a very small region of high radiation. On the adjacent marble and on the face of the downstream gradient magnet the rate is a bit above the nominal. These locations are away from the aisle, thus limiting exposure. 

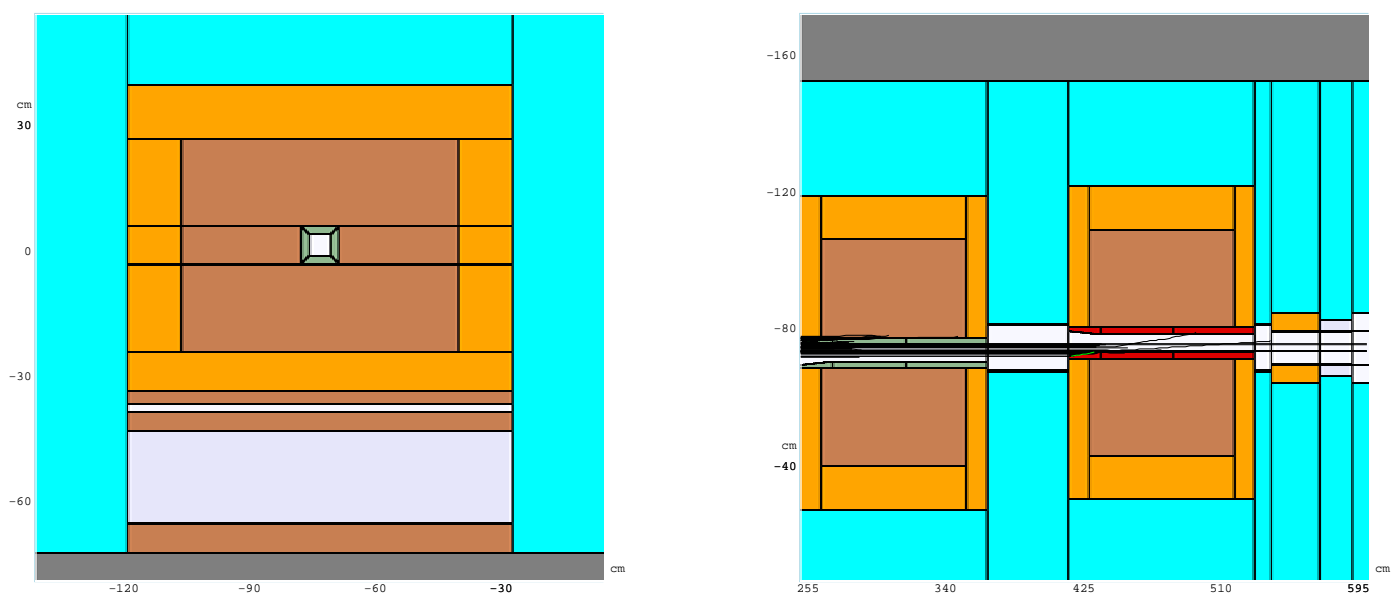

$\stackrel{\mathrm{X}}{\longrightarrow} \mathrm{Y}$

$\vec{P}_{\text {spect }}^{2}$

Figure 1: Schematic views of the proposed MI-8 collimators with marble shell: transverse (left) and plan with sampled particle tracks (right).

To re-scale the residual dose for the canonical 30-day / 1-day conditions used throughout the paper to other conditions, one may employ Table 2. Such a re-scaling depends on the location in the system (energy spectrum) and the material. Define conditions DO,D2,D2, and D3 by

- D0 as a residual dose for $T_{i r r}=30$ days and $T_{\text {cool }}=1$ day

- D1 as a residual dose for $T_{i r r}=1$ year and $T_{\text {cool }}=1$ hour,

- D2 as a residual dose for $T_{i r r}=1$ year and $T_{\text {cool }}=4$ hour.

- D3 as a residual dose for $T_{i r r}=20$ years and $T_{\text {cool }}=1$ hour,

Table 2: Radiation For Various Exposure/Cooldown Conditions

\begin{tabular}{|l|c|c|c|}
\hline Object & D1/D0 & D2/D0 & D3/D0 \\
\hline Stainless steel jaws & 2.4 & 2.1 & 2.8 \\
\hline Iron (collimator body and support at bottom) & 2.7 & 2.2 & 3.2 \\
\hline Marble shell & 8.1 & 2.6 & 8.7 \\
\hline
\end{tabular}

The main difference comes from a shorter cooling time, different for different materials with marble preferring longer ( $>4 \mathrm{hr}$ ) cooling than steel. 

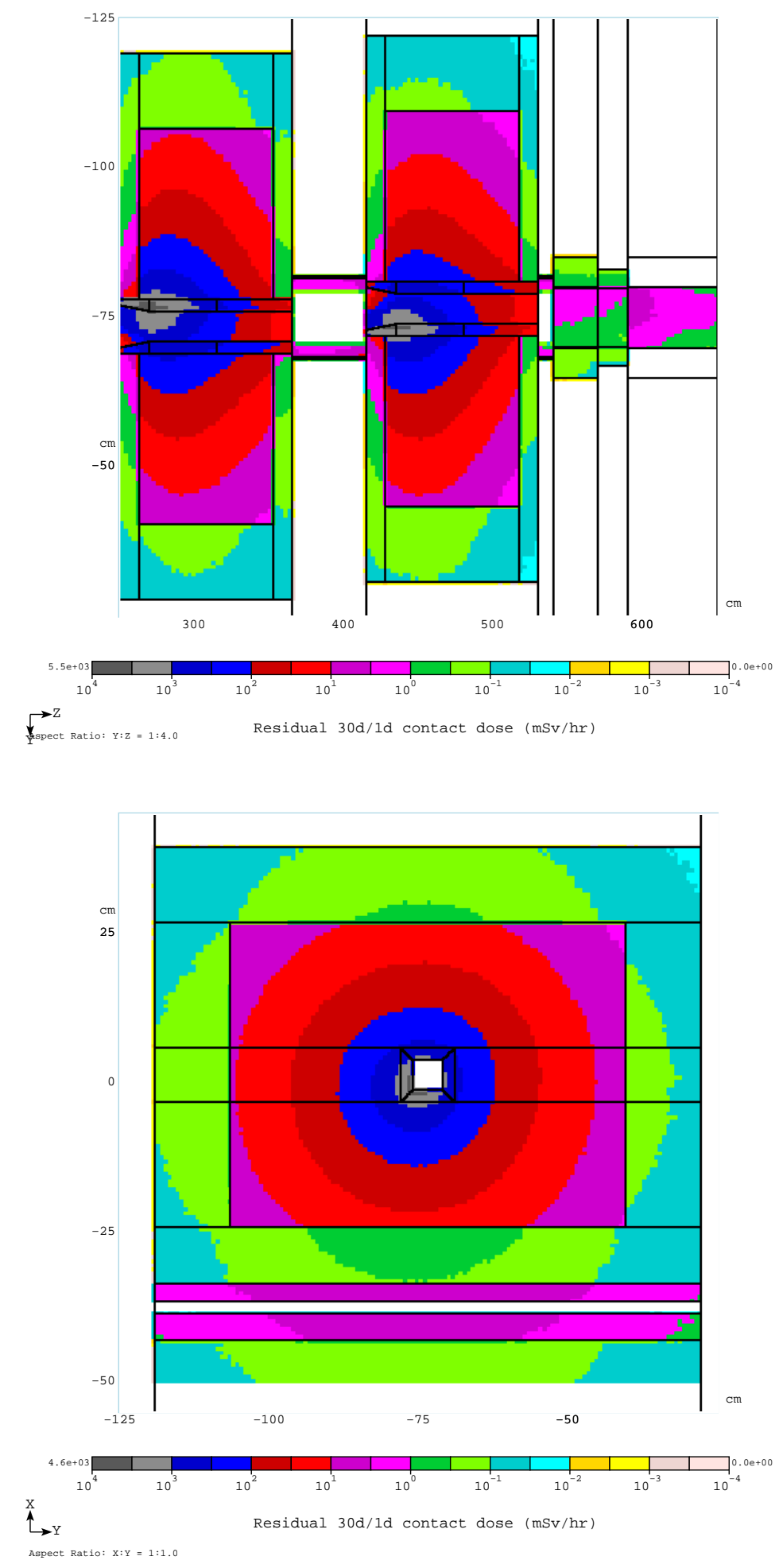

Figure 2: Residual dose isocontours in the collimators after 30 days of irradiation at $5 \times 10^{1} \mathrm{p} / \mathrm{s}$ and 1 day of cooling. See Table 2 for other irradiation/cooling scenarios. Top image shows plan view showing longitudinal profile along the system axis including the upstream collimator, bellows, downstream collimator, bellows, marble mask, BPM and upstream end of next magnet. Bottom image shows transverse profiles at the shower maximum location in the first (horizontal) collimator. For this simulation the first collimator cuts the bottom and left of the beam while the second one cuts the top and right. Note that $1 \mathrm{mSv} / \mathrm{hr}=100 \mathrm{mrem} / \mathrm{hr}$. 


\section{Energy Deposition}

The MARS 15 code simulates the interaction of protons in the beam halo with the collimators. Ionization energy, from the primary beam as well as all the secondary hadronic and electromagnetic shower particles, is simulated and the resulting energy deposition results recorded. The large taper of the MI8 collimator jaws ensures that the interacting particles will impact at a substantial angle (45 milliradians for particles parallel to the beam axis), enabling the collimator to capture a large fraction of the energy of incident particles (low out-scattering). The $1 / r$ halo distribution implies that most of the particles will strike near the downstream end of the tapered portion of the jaw. The shower develops in the stainless steel jaw, continuing with similar characteristics into the surrounding steel.

MARS15 results on energy deposition distributions in the collimators are presented in this section to feed the ANSYS thermal analysis [9]. Power density isocontours along the beam axis are presented in Fig. 3 for vertical and horizontal planes. Fig. 4 shows power density isocontours in a transverse plane at corresponding shower maxima in each collimator. Longitudinal distributions of power density in the hottest bottom stainless steel jaw and in the first layer of steel shielding immediately outside this jaw are shown in Fig. 5 for the first (horizontal) collimator. Vertical and horizontal profiles of power density at shower maxima are given in Figs. 6 and 7 for the horizontal and vertical collimators, respectively.
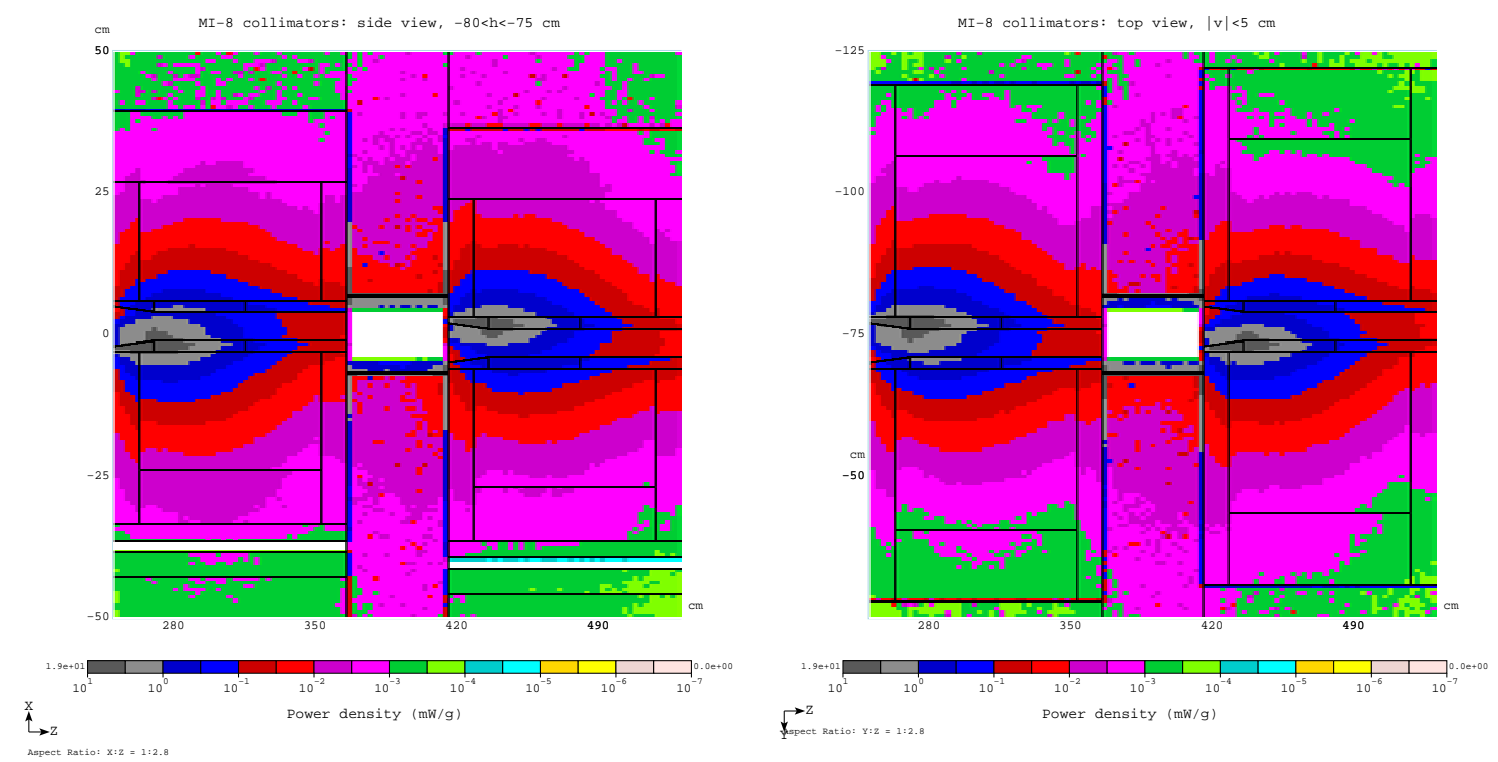

Figure 3: Power density isocontours along beam axis in vertical (left) and horizontal planes (right). 

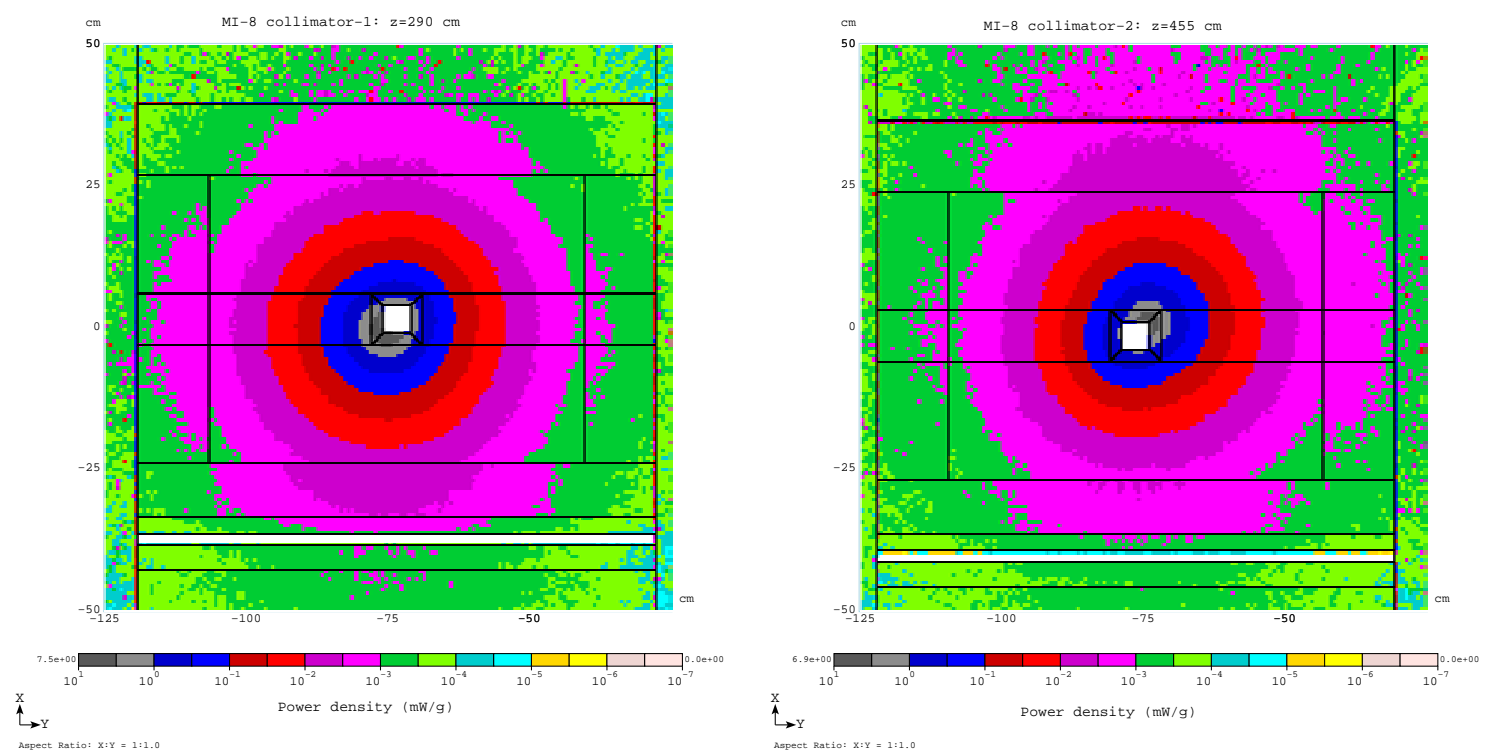

Figure 4: Power density isocontours at longitudinal maxima in upstream (left) and downstream (right) collimators.
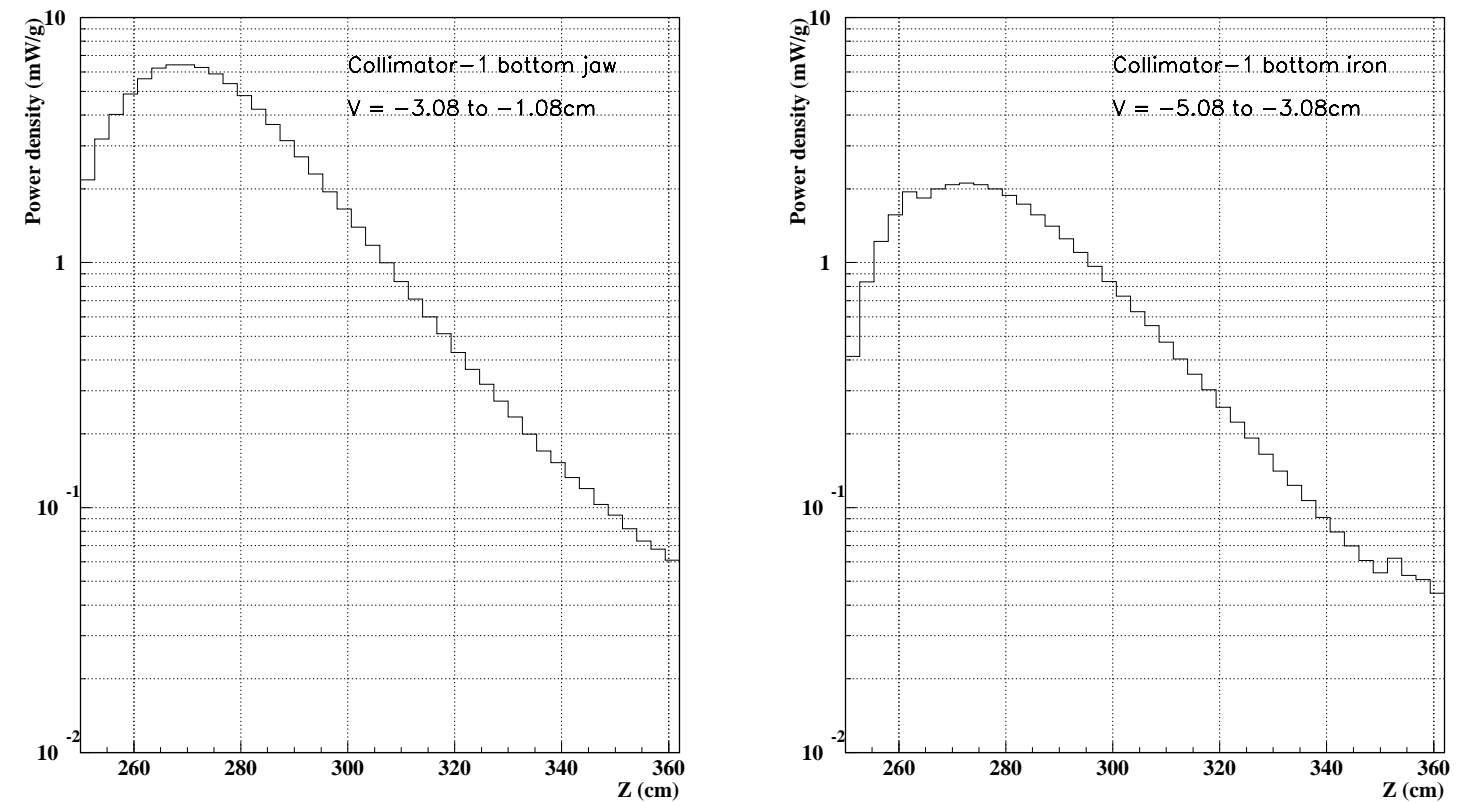

Figure 5: Longitudinal profile of peak power density in the stainless steel jaw (left) and in shielding iron immediately outside the jaw (right) for the horizontal collimator. 

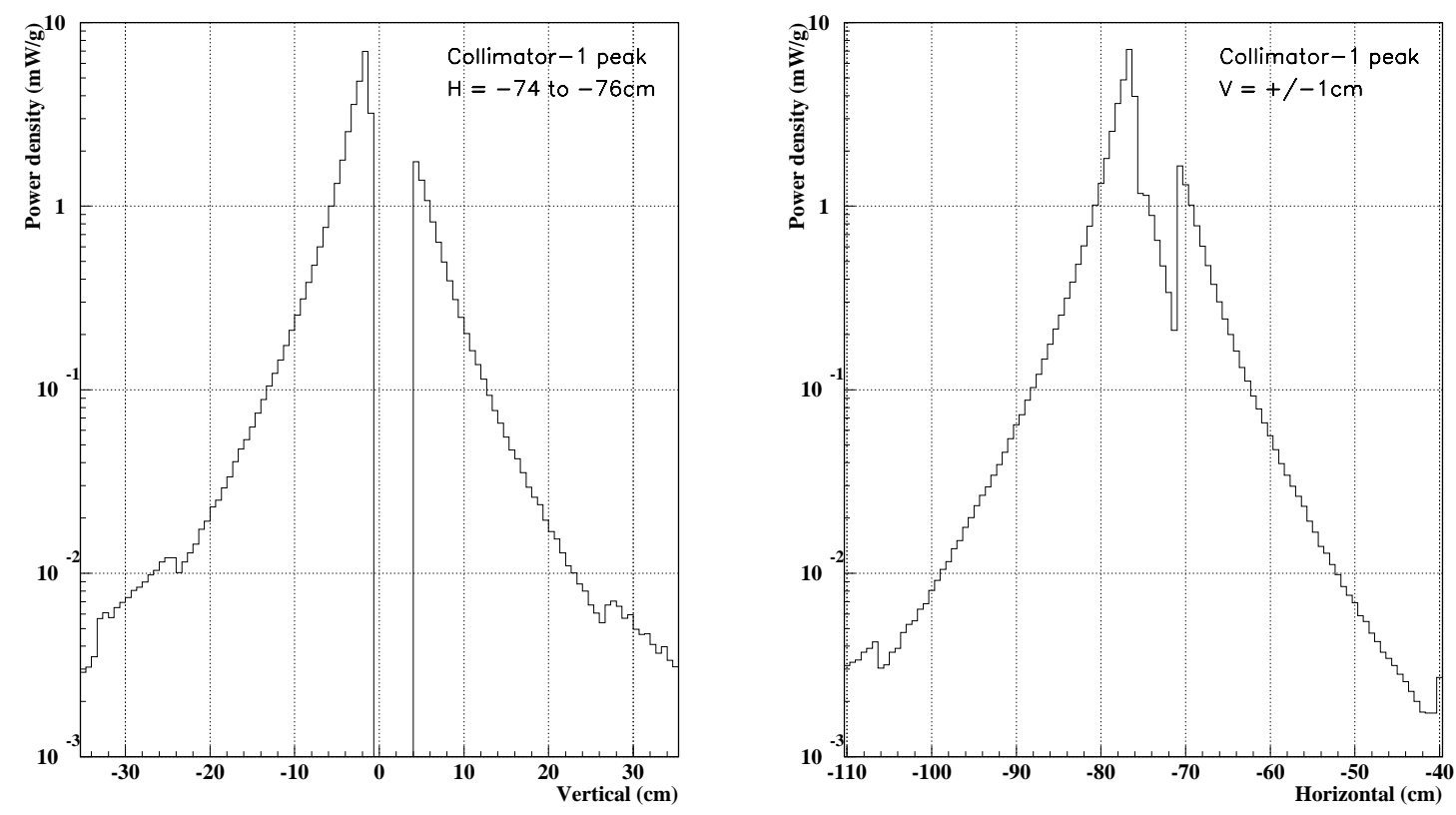

Figure 6: Vertical (left) and horizontal (right) profiles of power density at shower maximum in the horizontal collimator.
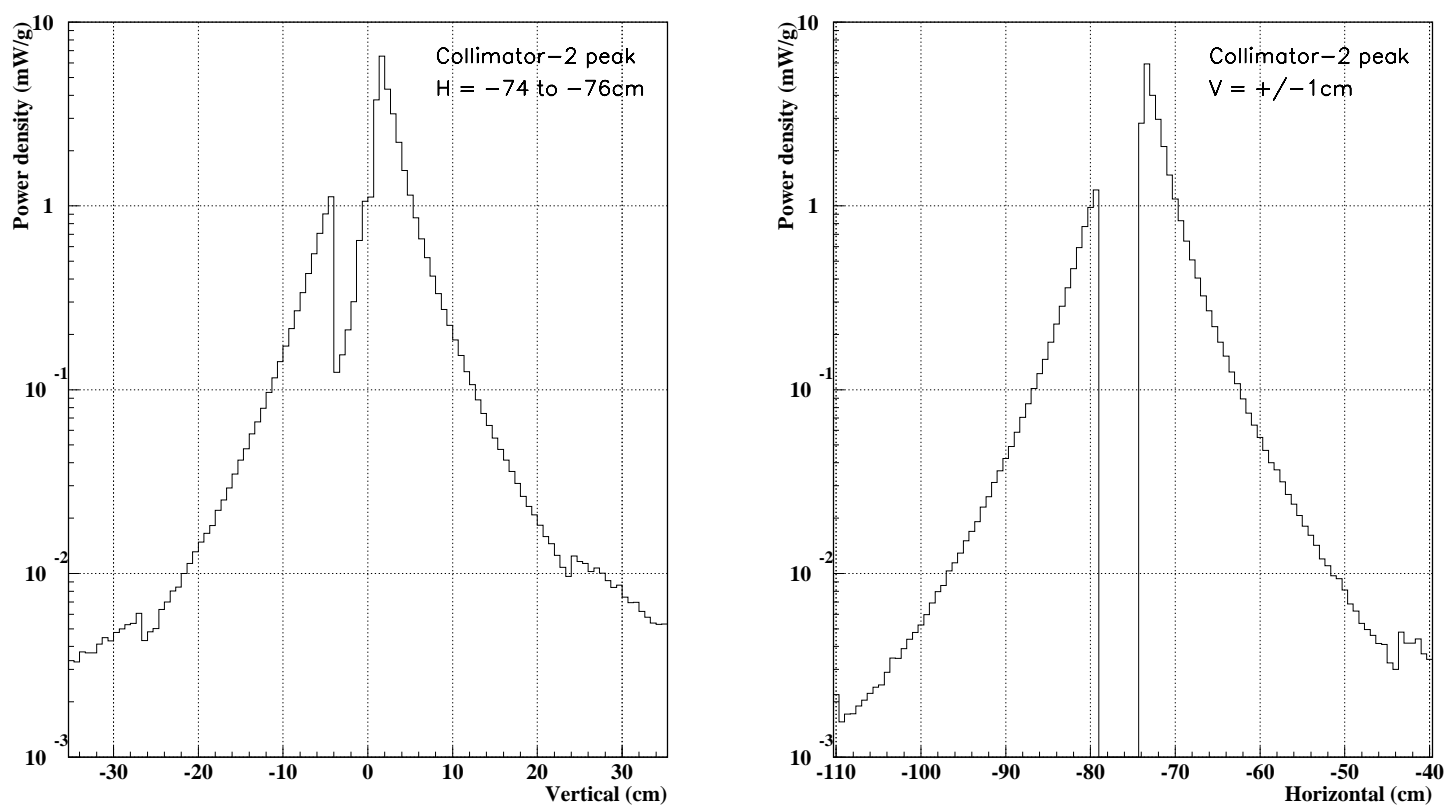

Figure 7: Vertical (left) and horizontal (right) profiles of power density at shower maximum in the vertical collimator. 


\section{Radiation Fields in Collimator Region}

A quite realistic MARS 15 model of the MI- 8 collimator region includes all the essential components. Besides a very detailed description of all the collimator parts, it contains all the beam pipes with transition sections, support plates, motors, downstream magnets, a realistic tunnel and a dirt outside. The prompt dose on the Earth surface above this region is well below the limits for unlimited occupancy area. The peak residual dose rates on contact after 30-day irradiation and 1-day cooling are as follows (in $\mathrm{mrem} / \mathrm{hr}$ ): outside marble surface (top, sides) $<50$; steel support plate - 1300; front face marble surface few hundred; motors - 600; floor - 200; ceiling - 150; near wall - 400; far wall (aisle side) - 100. For the 837A magnet, the peak doses on the mask, BPM and magnet itself are 6000,150 and $1500 \mathrm{mrem} / \mathrm{hr}$, respectively, with an iron mask, and $<100,170$ and $2500 \mathrm{mrem} / \mathrm{hr}$ with a marble mask. See Table 2 for other irradiation/cooling scenarios.

All these are the peak values found in calculations for the hottest (small) spots. Averaging over a larger area (a square foot as recommended by ES\&H), would reduce these numbers a few times on the components, and about by $50 \%$ on the floor, ceiling and walls. "A good-practice" design goal here is $<100 \mathrm{mrem} / \mathrm{hr}$, at one foot from the surface in the walkway (aisle). Most of the above values need to be further reduced by about a factor of two then. The tolerances are much higher on top and bottom. It seems that we are OK here.

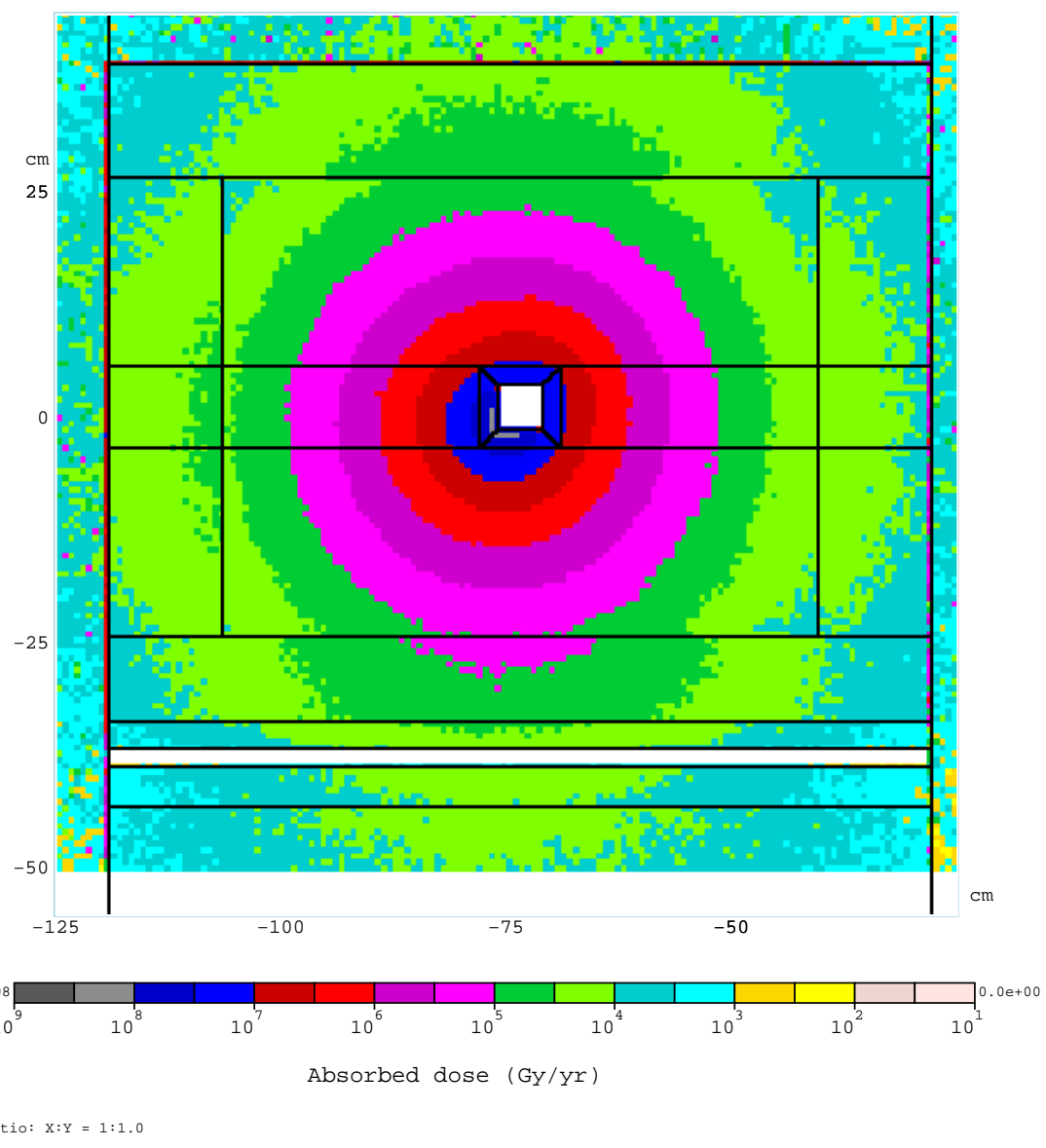

Figure 8: Annual absorbed dose transverse isocontours at the horizontal collimator shower maximum location. 
Isocontours of the absorbed dose accumulated over an operational year in the first (horizontal) collimator are shown in Fig. 8 for a shower maximum location at about $30 \mathrm{~cm}$ from the upstream end. These results can be directly used to estimate the collimator component lifetime. Although the dose in the collimator core is very high, the peak radiation levels at motors, cables and magnets are much lower: $4 \mathrm{Mrad} / \mathrm{yr}$ on motors, and 40 and $60 \mathrm{Mrad} / \mathrm{yr}$ on the 837A magnet with iron and marble masks, respectively.

Fig. 9 presents residual and absorbed dose transverse isocontours at the hottest (upstream) end of the 837A magnet behind the vertical collimator CV. The results are shown with a 1-foot long marble mask $\left(20 \times 20 \mathrm{~cm}^{2}\right)$ surrounding the beam pipe immediately downstream of the $\mathrm{CV}$ collimator. It reduces the radiation levels in the magnet by almost an order of magnitude. To feed the air activation analysis, hadron flux distributions in and around the collimators have been calculated (Fig. 10).
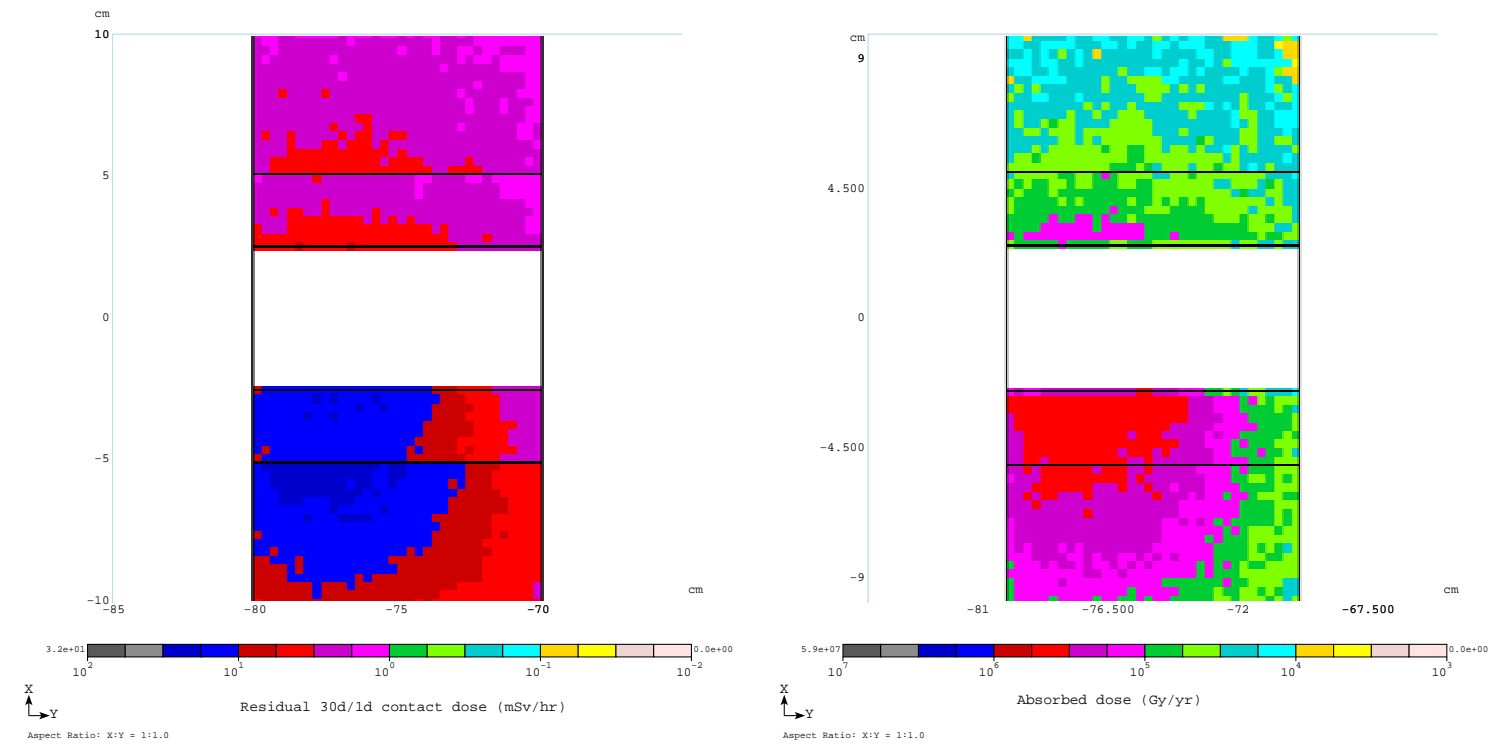

Figure 9: Residual dose transverse isocontours at the upstream end of the 837A magnet after 30 days of irradiation at $5 \times 10^{11} \mathrm{p} / \mathrm{s}$ and 1 day of cooling (left). Annual absorbed dose transverse isocontours in the upstream end of the 837A magnet (right). 

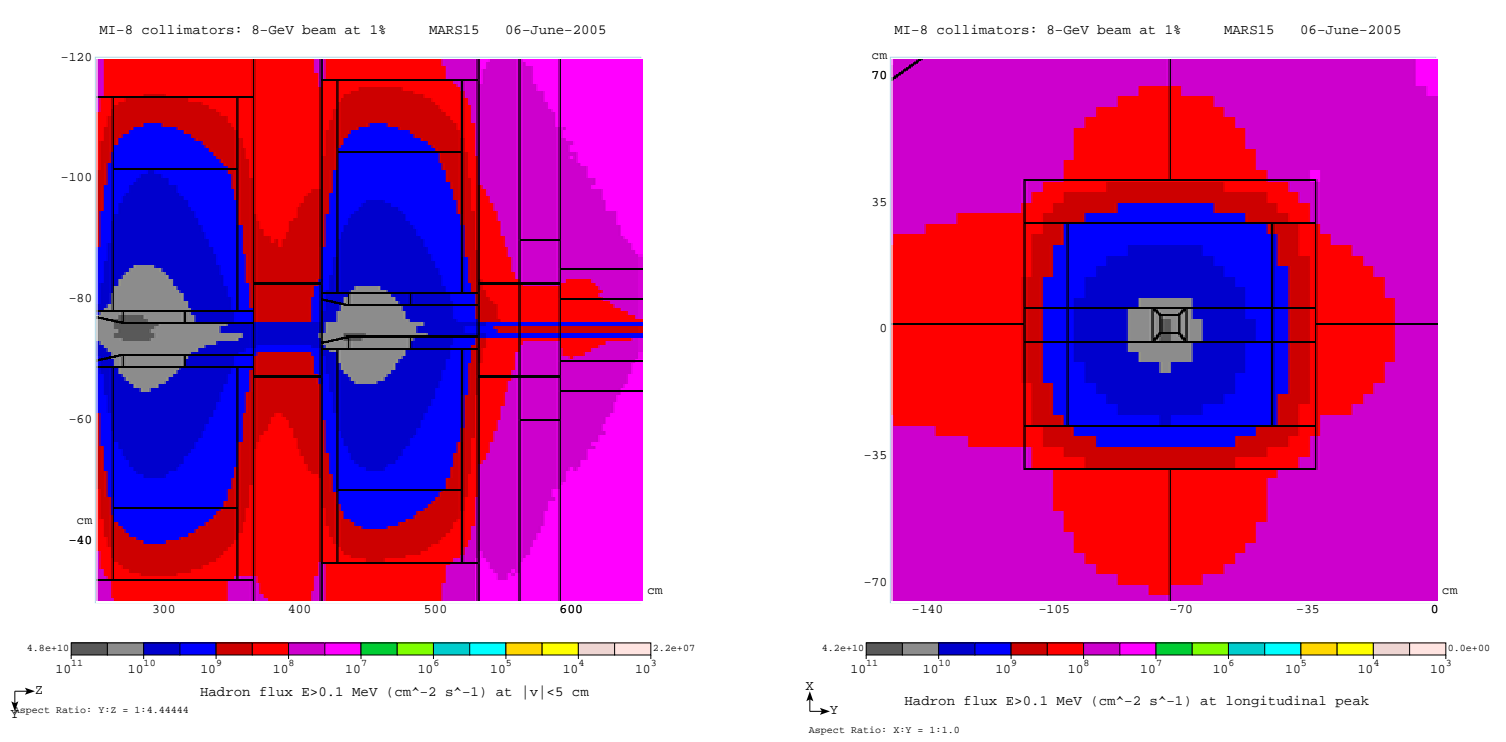

Figure 10: Longitudinal (left) and transverse (right) hadron flux in and near collimators.

\section{Sump Water Contamination}

Fermilab concentration model [10] relates ground and sump water activation to star density in the dirt containing these waters. Figs. 11 and 12 show star density isocontours in the collimation region. The current ES\&H Fermilab rule assumes star density averaging over a dirt volume that contains $99 \%$ of stars. For the region considered, it corresponds to $0<z<12 \mathrm{~m}$ averaging longitudinally (Fig. 11) and averaging over two meters of dirt immediately outside of the tunnel walls laterally (Fig. 12). Averaged over four sides - left, right, top and bottom - it gives us $\langle S\rangle=1040 \mathrm{~cm}^{-3} \mathrm{~s}^{-1}$, almost a factor of 4 below the limit for sump water. This is calculated for the $1 \%$ scraping scenario with $5 \times 10^{11} \mathrm{p} / \mathrm{s}$ loss at one pair of collimators or $\langle S\rangle=2.08 \times 10^{-9} \mathrm{~cm}^{-3} \mathrm{p}^{-1}$. Note that most water from 836 and 838 will appear at the MI- 8 SB SP17 sump pump but the limits are based on the star volume at a given location so these limits are not impacted by pump location.

\section{Conclusions}

The collimation system developed for the Fermilab MI-8 beamline will localize majority of beam loss in a predetermined 836B-837A region followed by a second pair of collimators downstream. The collimator/shielding units developed for a scraping rate of $1 \%$ (averaged over months of operation) and optimized via detailed MARS15 simulations, will provide adequate collimation, shielding and maintenance functionalities, assuring that prompt and residual radiation levels inside the tunnel, in sump water and above the dirt shielding are below the regulatory limits with a reasonable safety margin. Distributions of hadron fluxes calculated in the collimation region can be used as a source for air activation analysis.

\section{References}

[1] B.C. Brown, "Residual Radiation Hints for Aperture and Alignment Issues in the Main Injector", Fermilab-Beams-Doc-1382 (2004). 


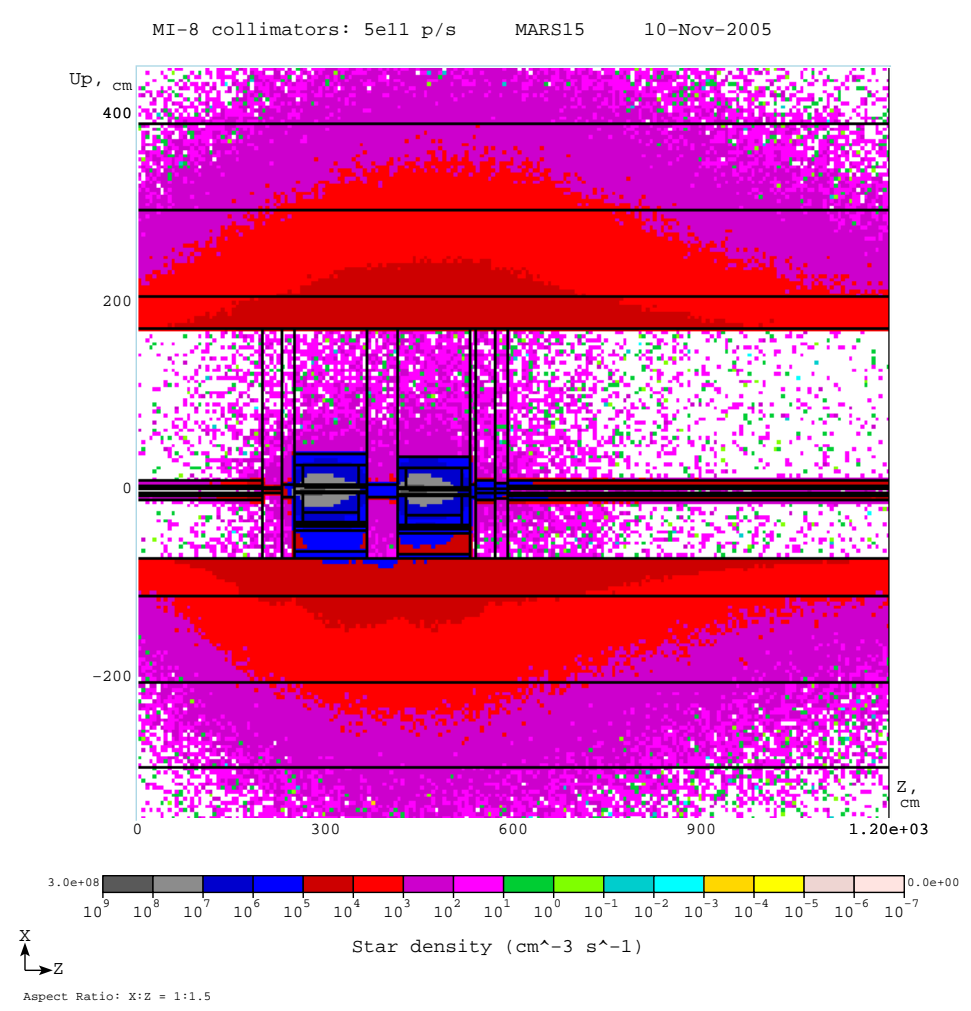

Figure 11: Star density isocontours in and near collimators: longitudinal profiles along the system axis.

[2] B.C. Brown, "MI8 Beamline Collimation Design”, Fermilab-Beams-Doc-1977 (2005).

[3] V. Sidorov, A. Legan, B.C. Brown, "Mechanical Design of MI8 Collimators", Fermilab-BeamsDoc-2287 (2006).

[4] N.V. Mokhov, "MARS15 Modeling of MI-8 Beamline Collimation”, Fermilab-Beams-Doc1826 (2005).

[5] N.V. Mokhov, “The Mars Code System User's Guide", Fermilab-FN-628 (1995); N.V. Mokhov, K.K. Gudima, C. James et al., "Recent enhancements to the MARS15 code", Radiation Protection Dosimetry, v. 116, pp. 99-103 (2005), Fermilab-Conf-04/053 (2004); http://www-ap.fnal.gov/MARS/.

[6] N.V. Mokhov et al, "Fermilab Booster Beam Collimation and Shielding", Proc. 2003 Part. Accel. Conf., Portland, May 2003, p. 1503. Also available as Fermilab-Conf-03-087.

[7] M.D. Church, A.I. Drozhdin, A. Legan, N.V. Mokhov, R.E. Reilly, "Tevatron Run-II Beam Collimation System", in 1999 Particle Accelerator Conf., IEEE Conference Proceedings, New York, 1999, pp. 56-58. Also available as Fermilab-Conf-99/059

[8] M. Coli. Geomechanical characterization of Carrara marble. In Sarkka and Eloranta, editors, Rock Mechanics a Challenge for Society, page 5. Swets \& Zeitlinger, 2001. ISBN 9026518218. 


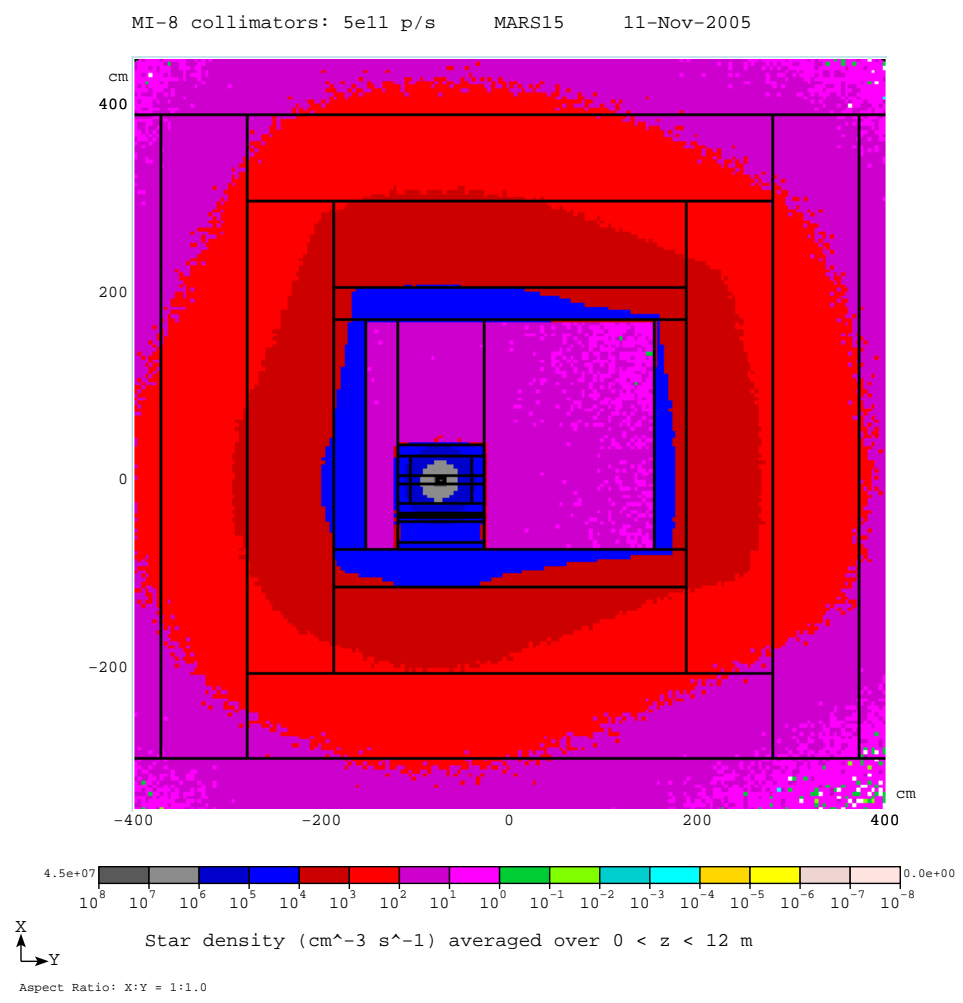

Figure 12: Star density isocontours in and near collimators: transverse profiles at the horizontal collimator shower maximum location.

[9] Alex Chen, Bruce C. Brown, and Zhijing Tang. "Thermal calculation for MI8 collimators". Beams Doc 2388, Fermilab, August 2006.

[10] Fermilab Radiological Control Manual, Article 236, http://www-esh.fnal.gov/FRCM/. 